\title{
Arithmetic coding with folds and unfolds
}

\author{
Richard Bird and Jeremy Gibbons \\ Programming Research Group, Oxford University \\ Wolfson Building, Parks Road, Oxford, OX1 3QD, UK
}

\section{Introduction}

Arithmetic coding is a method for data compression. Although the idea was developed in the 1970's, it wasn't until the publication of an "accessible implementation" [14] that it achieved the popularity it has today. Over the past ten years arithmetic coding has been refined and its advantages and disadvantages over rival compression schemes, particularly Huffman [9] and Shannon-Fano [5] coding, have been elucidated. Arithmetic coding produces a theoretically optimal compression under much weaker assumptions than Huffman and Shannon-Fano, and can compress within one bit of the limit imposed by Shannon's Noiseless Coding Theorem [13]. Additionally, arithmetic coding is well suited to adaptive coding schemes, both character and word based. For recent perspectives on the subject, see $[10,12]$.

The "accessible implementation" of [14] consisted of a 300 line C program, and much of the paper was a blow-by-blow description of the workings of the code. There was little in the way of proof of why the various steps in the process were correct, particularly when it came to the specification of precisely what problem the implementation solved, and the details of why the inverse operation of decoding was correct. This reluctance to commit to specifications and correctness proofs seems to be a common feature of most papers devoted to the topic. Perhaps this is not surprising, because the plain fact is that arithmetic coding is tricky. Nevertheless, our aim in these lectures is to provide a formal derivation of basic algorithms for coding and decoding.

Our development of arithmetic coding makes heavy use of the algebraic laws of folds and unfolds. Although much of the general theory of folds and unfolds is well-known, see $[3,6]$, we will need one or two novel results. One concerns a new pattern of computation, which we call streaming. In streaming, elements of an output list are produced as soon as they are determined. This may sound like lazy evaluation but it is actually quite different.

\section{Arithmetic coding, informally}

Arithmetic coding is simple in theory but, as we said above, tricky to implement in practice. The basic idea is to:

1. Break the source message into symbols, where a symbol is some logical grouping of characters (or perhaps just a single character). 
2. Associate each distinct symbol with a semi-open interval of the unit interval $[0 . .1)$.

3. Successively narrow the unit interval by an amount determined by the interval associated with each symbol in the message.

4. Represent the final interval by choosing some fraction within it.

We can capture the basic datatypes and operations in Haskell by defining

$$
\begin{aligned}
& \text { type Fraction }=\text { Ratio Integer } \\
& \begin{array}{ll}
\text { type Interval } & =(\text { Fraction }, \text { Fraction }) \\
\text { unit } & :: \text { Interval } \\
\text { unit } & =(0,1) \\
\text { within } & :: \text { Fraction } \rightarrow \text { Interval } \rightarrow \text { Bool } \\
\text { within } x(l, r) & =l \leq x \wedge x<r \\
\text { pick } & :: \text { Interval } \rightarrow \text { Fraction } \\
\text { pick }(l, r) & =(l+r) / 2
\end{array}
\end{aligned}
$$

Except where otherwise stated, we assume throughout that $0 \leq l<r \leq 1$ for every $(l, r)::$ Interval, so all intervals are subintervals of the unit interval. The code above gives a concrete implementation of pick, but all we really require is that

pick int within int

(We use underlining to turn a prefix function into an infix binary operator; this would be written 'within' in Haskell.)

\subsection{Narrowing}

The operation of narrowing takes two intervals $i$ and $j$ and returns a subinterval $k$ of $i$ such that $k$ is in the same relationship to $i$ as $j$ is to the unit interval:

$$
\begin{array}{ll}
(\triangleright) & :: \text { Interval } \rightarrow \text { Interval } \rightarrow \text { Interval } \\
(l, r) \triangleright(p, q) & =(l+(r-l) \times p, l+(r-l) \times q)
\end{array}
$$

Diagrammatically, we have:

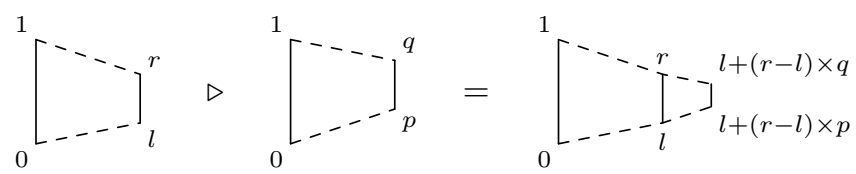

Exercise 1. Prove that $x \underline{\text { within }}\left(\right.$ int $_{1} \triangleright$ int $\left._{2}\right) \Rightarrow x \underline{\text { within }}$ int $_{1}$.

Exercise 2. Show that $\triangleright$ is associative with identity unit. Is $\triangleright$ commutative?

Exercise 3. Define an inverse $\triangleleft$ ('widen') of $\triangleright$ such that $\left(\right.$ int $\left._{1} \triangleright i n t_{2}\right) \triangleleft i n t_{1}=i n t_{2}$.

Exercise 4. Define the notion of the reciprocal $i^{-1}$ of an interval $i$, such that

$$
i \triangleright i^{-1}=\text { unit }=i^{-1} \triangleright i
$$

(The reciprocal of a sub-unit interval will in general not itself be a sub-unit.) Redefine widening in terms of narrowing and reciprocal. 


\subsection{Models}

In order to encode a message, each symbol has to be associated with a given interval. For our purposes, Model is an abstract type representing a finite mapping from Symbols to Intervals with associated functions:

$$
\begin{aligned}
& \text { encodeSym }:: \text { Model } \rightarrow \text { Symbol } \rightarrow \text { Interval } \\
& \text { decodeSym }:: \text { Model } \rightarrow \text { Fraction } \rightarrow \text { Symbol }
\end{aligned}
$$

We assume that the intervals associated with symbols do not overlap: for any $m::$ Model and $x::$ Fraction,

$$
s=\text { decodeSym } m x \equiv x \underline{\text { within }}(\text { encodeSym } m s)
$$

Rather than having a single fixed model for the whole message, we allow the possibility that the model can change as the message is read; such a scheme is called adaptive. For instance, one can begin with a simple model in which symbols are associated via some standard mapping with intervals of the same size, and then let the model adapt depending on the actual symbols read. Therefore we also assume the existence of a function

$$
\text { newModel }:: \text { Model } \rightarrow \text { Symbol } \rightarrow \text { Model }
$$

As long as the decoder performs the same adaptations as the message is reconstructed, the message can be retrieved. Crucially, there is no need to transmit the model with the message. The idea of an adaptive model is not just a useful refinement on the basic scheme, but also an essential component in the derivation of the final algorithms.

Exercise 5. Specify the stronger condition that the intervals associated with symbols partition the unit interval.

\subsection{Encoding}

Having defined the relevant datatypes and auxiliary operations we can now define arithmetic encoding, which is to compute encode $_{0} m$ unit, where

$$
\begin{array}{ll}
\begin{array}{ll}
\text { encode }_{0} \\
\text { encode }_{0} m \text { int }
\end{array} & :: \text { Model } \rightarrow \text { Interval } \rightarrow[\text { Symbol }] \rightarrow \text { Fraction } \\
\text { encodeSyms } \cdot \text { foldl }(\triangleright) \text { int } \cdot \text { encodeSyms } m \\
\text { encodeSyms } m \text { ss }=\text { unfoldr nextInt }(m, \text { ss }) \\
\text { nextInt }:: \text { Model },[\text { Symbol }]) \rightarrow \\
& \text { Maybe }(\text { Interval },(\text { Model },[\text { Symbol }])) \\
\text { nextInt }(m,[])= & \text { Nothing } \\
\text { nextInt }(m, s: s s)= & \text { Just }(\text { encodeSym } m s,(\text { newModel } m s, s s))
\end{array}
$$

The function encodeSyms $m$ uses the initial model $m$ to encode the symbols of the message as intervals. These intervals are then used to narrow the unit interval to some final interval from which some number is chosen. The code makes use of the standard Haskell higher-order operators foldl and unfoldr, which are discussed in more detail in the following section. 


\subsection{Decoding}

What remains is the question of how to perform the inverse operation of arithmetic decoding. Rather than give a program, we will give a non-executable specification. The function decode $0::$ Model $\rightarrow$ Interval $\rightarrow$ Fraction $\rightarrow[$ Symbol $]$ is specified by

$$
\text { ss } \underline{\text { begins }} \text { (decode } \text { de } \text { int (encode } m \text { int ss)) }
$$

for all $s s$, where $x s \underline{\text { begins }} y s$ if $y s=x s+x s^{\prime}$ for some $x s^{\prime}$. Thus decode $e_{0}$ is inverse to encode $e_{0}$ in the sense that it is required to produce the sequence of symbols that encode $_{0}$ encodes but is not required to stop after producing them. Termination is handled separately. Provided we record the number of symbols in the message, or ensure that it ends with a special end-of-message symbol that occurs nowhere else, we can stop the decoding process at the right point.

Exercise 6. The Haskell definition of begins :: Eq $\alpha \rightarrow[\alpha] \rightarrow[\alpha] \rightarrow$ Bool is

$$
\begin{array}{ll}
{[] \underline{\text { begins }} y s} & =\text { True } \\
(x: x s) \underline{\text { begins }}[] & =\text { False } \\
(x: x s) \underline{\text { begins }}(y: y s) & =(x==y \wedge x \text { s } \underline{\text { beg } \underline{\text { ins }} y s)}
\end{array}
$$

What is the value of [] $\underline{\text { begins }} \perp$ ?.

Exercise 7. What are the advantages and disadvantages of the two schemes (returning the length of the message, or making use of a special end-of-message symbol) for determining when to stop decoding?

\subsection{Remaining refinements}

Simple though encode $_{0}$ is, it will not suffice in a viable implementation and this is where the complexities of arithmetic coding begin to emerge. Specifically:

- we really want an encoding function that returns a list of bits (or bytes) rather than a number, not least because -

- for efficiency both in time and space, encoding should produce bits as soon as they are known (this is known as incremental transmission, or streaming);

- consequently, decoding should be implemented as a function that consumes bits and produces symbols, again in as incremental a manner as possible;

- for efficiency both in time and space, we should replace computations on fractions (pairs of arbitrary precision integers) with computations on fixedprecision integers, accepting that the consequent loss of accuracy will degrade the effectiveness of compression;

- we have to choose a suitable representation of models.

All of the above, except the last, will be addressed in what follows. We warn the reader now that there is a lot of arithmetic in arithmetic coding, not just the arithmetic of numbers, but also of folds and unfolds. 


\section{Folds and unfolds}

Let us now digress a little to recall some of the theory of folds and unfolds. We will return to and augment our understanding of these operators in subsequent sections.

The higher-order operator foldl iterates over a list, from left to right:

$$
\begin{array}{ll}
\text { foldl } & ::(\beta \rightarrow \alpha \rightarrow \beta) \rightarrow \beta \rightarrow[\alpha] \rightarrow \beta \\
\text { foldl } f e[] & =e \\
\text { foldl } f e(x: x s) & =\text { foldl } f(f \text { e } x) \text { xs }
\end{array}
$$

Thus, writing $f$ as an infix operator $\oplus$, we have

$$
\text { foldl }(\oplus) e[x, y, z]=((e \oplus x) \oplus y) \oplus z
$$

Dually, the higher-order operator foldr iterates over a list, from right to left:

$$
\begin{array}{ll}
\text { foldr } & ::(\alpha \rightarrow \beta \rightarrow \beta) \rightarrow \beta \rightarrow[\alpha] \rightarrow \beta \\
\text { foldr } f e[] & =e \\
\text { foldr } f e(x: x s) & =f x(\text { foldr } f \text { e } x s)
\end{array}
$$

Thus, foldr $(\oplus) e[x, y, z]=x \oplus(y \oplus(z \oplus e))$. The crucial fact about foldr is the following universal property: for a strict function $h$ we have

$$
h=\text { foldr } f e \equiv h[]=e \wedge h(x: x s)=f x(h x s)
$$

There is a close relationship between foldl and foldr, captured in part by the following two theorems. As the names of the theorems suggest, we are not telling the whole story here.

Theorem 8 (First Duality Theorem [3]). If $f$ is associative with unit $e$, then foldl $f$ e $x s=$ foldr $f$ e xs for all finite lists xs.

Theorem 9 (Third Homomorphism Theorem [7]). If both $h=$ foldl $f_{1} e$ and $h=$ foldr $f_{2} e$, then there is an associative $f$ with unit $e$ such that $h=$ foldr $f e$.

From Theorem 8 and Exercise 2, we have

$$
\text { foldl }(\triangleright) \text { unit }=\text { foldr }(\triangleright) \text { unit }
$$

So why don't we use the arguably more familiar foldr to express arithmetic coding? The answer lies in the the following lemma, which turns out to be an essential step in obtaining a program for decoding:

\section{Lemma 10.}

$$
\text { foldl }(\triangleright) \text { int } \cdot \text { encodeSyms } m=\text { snd } \cdot \text { foldl step }(m, \text { int })
$$

where

$$
\text { step }(m, \text { int }) s=(\text { newModel } m s, \text { int } \triangleright \text { encodeSym } m s)
$$


This lemma shows how two computations, namely turning the sequence of symbols into a sequence of intervals and then combining that sequence of intervals into a single interval, can be fused into one. Fusion is perhaps the single most important general idea for achieving efficient computations. There is no equivalent lemma if we replace foldl by foldr.

Exercise 11. Using the universal property, prove the fusion theorem for foldr: provided $h$ is a strict function, $h e=e^{\prime}$ and $h\left(\begin{array}{lll}f & x & z\end{array}\right)=f^{\prime} x(h x)$ for every $x$ and $z$, we have $h \cdot$ foldr $f e=$ foldr $f^{\prime} e^{\prime}$.

Exercise 12. By defining map as an instance of foldr, prove map fusion:

$$
\text { foldr } f e \cdot \operatorname{map} g=\text { foldr }(f \cdot g) e
$$

Exercise 13. Why don't the universal property and the fusion theorem for foldr hold for non-strict $h$ ? Does the First Duality Theorem hold for infinite or partial lists?

Exercise 14. Suppose that $(x \oplus y) \ominus x=y$ for all $x$ and $y$. Prove that

$$
\text { foldl }(\ominus)(\text { foldr }(\oplus) x \text { ys }) \text { ys }=x
$$

for all $x$ and finite lists $y s$.

Exercise 15. 'Parallel loops' may also be fused into one: if

$$
h x s=\left(\text { foldr } f_{1} e_{1} x s, \text { foldr } f_{2} e_{2} x s\right)
$$

then $h=$ foldr $f\left(e_{1}, e_{2}\right)$, where $f x\left(z_{1}, z_{2}\right)=\left(\begin{array}{lllll}f_{1} & x & z_{1}, f_{2} & x & z_{2}\end{array}\right)$. For example,

$$
\text { average }=\text { uncurry div } \cdot \text { sumlength }
$$

where sumlength $x s=($ sum $x$, length $x s)$, and sumlength can be written with a single foldr. Parallel loop fusion is sometimes known as the "Banana Split Theorem' (because, in days of old, folds were often written using "banana" brackets; see, for example, [4]). Prove the theorem, again using the universal property of foldr.

Exercise 16. The function foldl can be expressed in terms of foldr:

$$
\text { foldl } f=\text { flip }(\text { foldr }(\operatorname{comp} f) \text { id) where comp } f x u=u \cdot \text { flip } f x
$$

Verify this claim, and hence (from the universal property of foldr) derive the following universal property of foldl: for $h$ strict in its second argument,

$$
h=f o l d l f \equiv h e[]=e \wedge h e(x: x s)=h(f \text { ex } x) x s
$$




\subsection{Unfolds}

To describe unfolds first recall the Haskell standard type Maybe:

$$
\text { data Maybe } \alpha=\text { Just } \alpha \mid \text { Nothing }
$$

The function unfoldr is defined by

$$
\begin{aligned}
\text { unfoldr }::(\beta \rightarrow \text { Maybe }(\alpha, \beta)) \rightarrow \beta \rightarrow[\alpha] \\
\text { unfoldr } f \quad b=\text { case } f b \text { of } \\
\text { Just }\left(a, b^{\prime}\right) \rightarrow a: \text { unfoldr } f b^{\prime} \\
\text { Nothing } \rightarrow[]
\end{aligned}
$$

For example, the standard Haskell prelude function enumFrom To is very nearly given by curry (unfoldr next), where

$$
\text { next }(a, b)=\text { if } a \leq b \text { then Just }(a,(\text { succ } a, b)) \text { else Nothing }
$$

(Only 'very nearly' because membership of the type class Enum does not actually imply membership of Ord in Haskell; the comparison is done instead by using fromEnum and comparing the integers.)

The Haskell Library Report [2] states:

The unfoldr function undoes a foldr operation....

$$
\text { unfoldr } f^{\prime}(\text { foldr } f z x s)=x s
$$

if the following holds:

$$
\begin{aligned}
f^{\prime}(f x y) & =\text { Just }(x, y) \\
f^{\prime} z & =\text { Nothing }
\end{aligned}
$$

That's essentially all the Report says on unfolds! We will have more to say about them later on.

\subsection{Hylomorphisms}

One well-known pattern involving folds and unfolds is that of a hylomorphism [11], namely a function $h$ whose definition takes the form

$$
h=\text { foldr } f e \cdot \text { unfoldr } g
$$

The two component computations have complementary structures and they can be fused into one:

$$
\begin{aligned}
& h z=\text { case } g z \text { of } \\
& \text { Nothing } \rightarrow e \\
& \text { Just }\left(x, z^{\prime}\right) \rightarrow f x\left(h z^{\prime}\right)
\end{aligned}
$$

This particular rule is known as deforestation because the intermediate data structure (in this case a list, but in a more general form of hylomorphism it could be a tree) is removed. 


\section{Producing bits}

Let us now return to arithmetic coding. As we noted above, we would like encoding to return a list of bits rather than a number. To achieve this aim we replace the function pick :: Interval $\rightarrow$ Fraction by two functions

$$
\begin{aligned}
& \text { type Bit }=\text { Int } \quad--\quad 0 \text { and } 1 \text { only } \\
& \text { toBits }:: \text { Interval } \rightarrow[\text { Bit }] \\
& \text { fromBits }::[\text { Bit }] \rightarrow \text { Fraction }
\end{aligned}
$$

such that pick $=$ fromBits $\cdot$ toBits. Equivalently, for all intervals int, we require

$$
\text { fromBits (toBits int) within int }
$$

The 'obvious' choices here are to let toBits $(l, r)$ return the shortest binary fraction $x$ satisfying $l \leq x<r$, and fromBits return the value of the binary fraction. Thus, fromBits $=$ foldr pack 0 , where pack $b x=(b+x) / 2$. However, as Exercises 25 and 26 explain, we reject the obvious definitions and take instead

$$
\begin{aligned}
\text { fromBits } & =\text { foldr pack }(1 / 2) \\
\text { toBits } & =\text { unfoldr nextBit }
\end{aligned}
$$

where

$$
\begin{array}{ll}
\text { nextBit } & :: \text { Interval } \rightarrow \text { Maybe }(\text { Bit }, \text { Interval }) \\
\text { nextBit }(l, r) & \\
\mid r \leq 1 / 2 & =\text { Just }(0,(2 \times l, 2 \times r)) \\
1 / 2 \leq l & =\text { Just }(1,(2 \times l-1,2 \times r-1)) \\
\mid \text { otherwise } & =\text { Nothing }
\end{array}
$$

Exercise 17. Give an equivalent definition of nextBit in terms of narrowing by non-sub-unit intervals.

We leave it as an exercise to show

$$
\text { foldr pack }(1 / 2) \text { bs = foldr pack } 0 \text { (bs }+[1])
$$

Thus fromBits bs returns the binary fraction obtained by adding a final 1 to the end of $b s$. The definition of toBits has a simple reading: if $r \leq 1 / 2$, then the binary expansion of any fraction $x$ such that $l<x<r$ begins with 0 ; and if $1 / 2 \leq l$, the expansion of $x$ begins with 1 . In the remaining case $l<1 / 2<r$ the empty sequence is returned.

Proposition 18. length $($ toBits $(l, r)) \leq-\log _{2}(r-l)$

In particular, toBits always yields a finite list given a non-empty interval.

Proof. The function toBits applied to an interval of width greater than a half yields the empty sequence of bits:

$$
0 \leq l<r \leq 1 \wedge 1 / 2<r-l \Rightarrow l<1 / 2<r
$$

Moreover, each iteration of nextBit doubles the width of the interval. So if $1 / 2^{n+1}<r-l \leq 1 / 2^{n}$ or, equivalently, $n \leq-\log _{2}(r-l)<n+1$, then termination is guaranteed after at most $n$ bits have been produced. 
Proposition 19. fromBits (toBits int) within int

Proof. The function pick $=$ fromBits $\cdot$ toBits is a hylomorphism, so we obtain

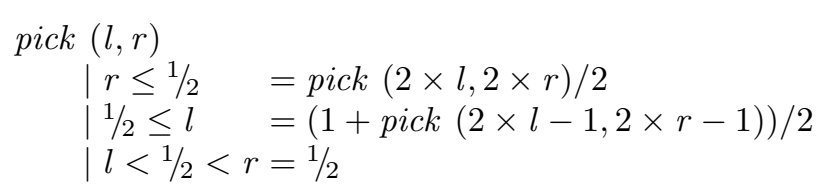

The proof now follows by appeal to fixpoint induction.

Exercise 20. Show that foldr pack $(1 / 2)$ bs $=$ foldr pack $0(b s+[1])$.

Exercise 21. Show that

$$
\begin{array}{ll}
(2 \times l, 2 \times r) & =(0,1 / 2) \triangleleft(l, r) \\
(2 \times l-1,2 \times r-1) & =(1 / 2,1) \triangleleft(l, r)
\end{array}
$$

Exercise 22. Show that

$$
\begin{gathered}
\text { fromBits bs = mean }(\text { foldr pack } 0 \text { bs, foldr pack } 1 \text { bs }) \\
\text { where mean }(x, y)=(x+y) / 2
\end{gathered}
$$

Exercise 23. Show that

$$
\begin{gathered}
\text { (foldr pack } 0 \text { bs, foldr pack } 1 \text { bs })=\text { foldl }(\triangleright) \text { unit (map encodeBit bs) } \\
\text { where encodeBit } b=(b / 2,(b+1) / 2)
\end{gathered}
$$

Exercise 24. One might expect toBits $(l, r)$ to yield the shortest binary fraction within $[l . . r)$, but in fact it does not. What definition does?

Exercise 25. The reason we do not use the shortest binary fraction as the definition of toBits is that the streaming condition of Section 5.1 fails to hold with this definition. After studying that section, justify this remark.

Exercise 26. Since we are using intervals that are closed on the left, one might expect that guard in the second clause of nextBit would be $1 / 2<l$. However, with this definition of fromBits, the result of Exercise 42 in Section 7 fails to hold. After studying that section, justify this remark.

\subsection{Summary of first refinement}

Drawing together the results of this section, we define

$$
\begin{aligned}
& \text { encode }_{1} \quad:: \text { Model } \rightarrow \text { Interval } \rightarrow[\text { Symbol }] \rightarrow[\text { Bit }] \\
& \text { encode }_{1} \text { m int }=\text { toBits } \cdot \text { foldl }(\triangleright) \text { int } \cdot \text { encodeSyms } m
\end{aligned}
$$

The new version of encoding yields a bit sequence rather than a fraction. However, execution of encode ${ }_{1}$ still consumes all its input before delivering any output. Formally, encode $e_{1} m s s=\perp$ for all partial or infinite lists $s s$. Can we do better? 


\section{$5 \quad$ Streaming}

The function encode $e_{1}$ consists of an unfoldr after a foldl. Even under lazy evaluation, the foldl consumes all its input before the unfoldr can start producing output. For efficiency, we would prefer a definition that is capable of yielding some output as soon as possible.

To this end, we introduce a new higher-order operator stream, which alternates between production and consumption. This function has type

$$
\begin{aligned}
\text { stream }:: & (\text { state } \rightarrow \text { Maybe }(\text { output }, \text { state })) \rightarrow \\
& (\text { state } \rightarrow \text { input } \rightarrow \text { state }) \rightarrow \\
& \text { state } \rightarrow[\text { input }] \rightarrow[\text { output }]
\end{aligned}
$$

and is defined by

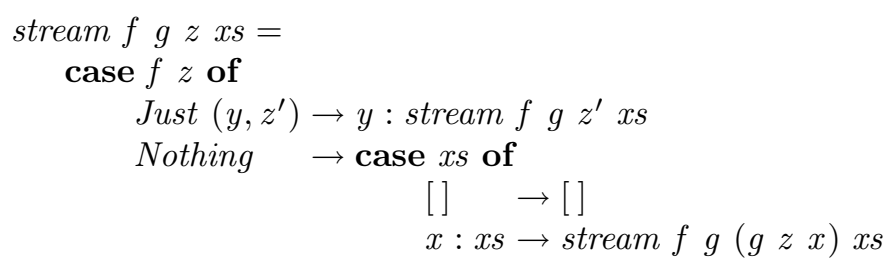

The function stream describes a process that alternates between producing output and consuming input. Starting in state $z$, control is initially passed to the producer function $f$, which delivers output until no more can be produced. Control is then passed to the consumer process $g$, which consumes the next input $x$ and delivers a new state. The cycle then continues until the input is exhausted.

Exercise 2\%. Define a variant stream that alternates between production and consumption but hands control to the consumer process first.

\subsection{The Streaming Theorem}

The relationship between stream and folds and unfolds hinges on the following definition:

Definition 28. The streaming condition for $f$ and $g$ is

$$
f z=\operatorname{Just}\left(y, z^{\prime}\right) \Rightarrow f(g z x)=\operatorname{Just}\left(y, g z^{\prime} x\right)
$$

for all $z, y, z^{\prime}$ and $x$.

The streaming condition states very roughly that $f$ is invariant under $g$. By induction we can then conclude that $f$ is invariant under repeated applications of $g$; this is the content of the following lemma:

Lemma 29. If the streaming condition holds for $f$ and $g$, then

$$
f z=\operatorname{Just}\left(y, z^{\prime}\right) \Rightarrow f(\text { foldl } g z x s)=\operatorname{Just}\left(y, \text { foldl } g z^{\prime} x s\right)
$$

for all $z, y, z^{\prime}$ and finite lists xs. 
Proof. The proof is by induction on $x s$ :

Case []: Immediate.

Case $x$ : xs: Assume $f z=$ Just $\left(y, z^{\prime}\right)$, so by the streaming condition we have $f(g z x)=$ Just $\left(y, g z^{\prime} x\right)$. Now we reason

$$
\begin{aligned}
& f(\text { foldl } g z(x: x s)) \\
= & \quad \text { definition of foldl }\} \\
& f(\text { foldl } g(g z x) x s) \\
= & \quad \text { induction }\} \\
& \text { Just }\left(y, \text { foldl } g\left(g z^{\prime} x\right) x s\right) \\
= & \text { ddefinition of foldl }\} \\
& \text { Just }\left(y, \text { foldl } g z^{\prime}(x: x s)\right)
\end{aligned}
$$

Now we come to the crunch.

Theorem 30. If the streaming condition holds for $f$ and $g$, then

$$
\text { unfoldr } f(\text { foldl } g \text { z xs })=\text { stream } f g z x s
$$

for all $z$ and all finite lists xs.

The proof of Theorem 30 uses the following lemma, which states how to prove that two potentially infinite lists are equal (see $[3, \S 9.3]$ ).

Lemma 31. Define approx by

$$
\begin{aligned}
& \text { approx }:: \text { Integer } \rightarrow[\alpha] \rightarrow[\alpha] \\
& \text { approx }(n+1)[]=[] \\
& \text { approx }(n+1)(x: x s)=x: \text { approx } n x s
\end{aligned}
$$

Then two arbitrary lists xs and ys are equal iff approx $n x s=$ approx $n$ ys for all $n$.

Proof (of Theorem 30). We use a double induction on $n$ and $x s$ to show that, provided that the streaming condition holds for $f$ and $g$,

$$
\text { approx } n \text { (unfoldr } f(\text { foldl } g z x s))=\operatorname{approx} n \text { (stream } f g z x s)
$$

for all $n, z$ and finite lists $x s$. The first step is case analysis on $n$.

Case 0: Immediate since approx $0 x s=\perp$ for any $x s$.

Case $n+1$ : In this case we perform an analysis on $f z$ :

Subcase $f z=$ Just $\left(y, z^{\prime}\right)$ : We reason

$$
\begin{aligned}
& \text { approx }(n+1)(\text { unfoldr } f(\text { foldl } g z x s)) \\
= & \quad \text { applying Lemma } 29\} \\
& \text { approx }(n+1)\left(y: \text { unfoldr } f\left(\text { foldl } g z^{\prime} x s\right)\right) \\
= & \{\text { definition of approx }\} \\
& y: \text { approx } n\left(\text { unfoldr } f\left(\text { foldl } g z^{\prime} x s\right)\right) \\
= & \{\text { induction }\} \\
& \left.y: \text { approx } n \text { (stream } f g z^{\prime} x s\right) \\
= & \{\text { definition of approx }\} \\
& \text { approx }(n+1)\left(y: \text { stream } f g z^{\prime} x s\right) \\
= & \{\text { definition of stream }\} \\
& \text { approx }(n+1)(\text { stream } f g z x s)
\end{aligned}
$$


Subcase $f z=$ Nothing: Now we need a case analysis on $x s$. The case of the empty list is immediate since both sides reduce to []. In the remaining case we reason

$$
\begin{aligned}
& \text { approx }(n+1)(\text { unfoldr } f(\text { foldl } g z(x: x s))) \\
= & \{\text { definition of foldl }\} \\
& \text { approx }(n+1)(\text { unfoldr } f(\text { foldl } g(g z x) x s)) \\
= & \{\text { induction }\} \\
& \text { approx }(n+1)(\text { stream } f g(g z x) x s) \\
= & \{\text { definition of stream }\} \\
& \text { approx }(n+1)(\text { stream } f g z(x: x s))
\end{aligned}
$$

This completes the induction and the proof.

Exercise 32. Show that the streaming condition holds for unCons and snoc, where

$$
\begin{array}{ll}
\text { unCons }[] & =\text { Nothing } \\
\text { unCons }(x: x s) & =\text { Just }(x, x s) \\
\text { snoc } x \text { xs } & =x s+[x]
\end{array}
$$

Exercise 33. What happens to the streaming theorem for partial or infinite lists?

Exercise 34. Recall that

$$
\begin{array}{ll}
\text { nextBit } & :: \text { Interval } \rightarrow \text { Maybe }(\text { Bit, Interval) } \\
\text { nextBit }(l, r) & \\
\mid r \leq 1 / 2 & =\text { Just }(0,(0,2) \triangleright(l, r)) \\
\mid 1 / 2 \leq l & =\text { Just }(1,(-1,1) \triangleright(l, r)) \\
\mid l<1 / 2<r & =\text { Nothing }
\end{array}
$$

Show that streaming condition for nextBit and $\triangleright$ follows from associativity of $\triangleright$ (Exercise 2) and the fact that int $t_{1} \triangleright i n t_{2}$ is contained in $i n t_{1}$ (Exercise 1).

\subsection{Summary of second refinement}

At the end of Section 4.1, we had

$$
\begin{aligned}
& \text { encode }_{1} \quad:: \text { Model } \rightarrow \text { Interval } \rightarrow[\text { Symbol }] \rightarrow[\text { Bit }] \\
& \text { encode }_{1} \text { m int }=\text { unfoldr nextBit } \cdot \text { foldl }(\triangleright) \text { int } \cdot \text { encodeSyms } m
\end{aligned}
$$

Since Exercise 34 established the streaming condition for nextBit and $\triangleright$, we can define

$$
\begin{aligned}
& \text { encode }_{2} \quad:: \text { Model } \rightarrow \text { Interval } \rightarrow[\text { Symbol }] \rightarrow[\text { Bit }] \\
& \text { encode }_{2} m \text { int }=\text { stream nextBit }(\triangleright) \text { int } \cdot \text { encodeSyms } m
\end{aligned}
$$

Although encode $_{1} \neq$ encode $_{2}$, the two functions are equal on all finite symbol sequences, which is all we require. 


\section{Decoding and stream inversion}

The function decode $2::$ Model $\rightarrow$ Interval $\rightarrow[$ Bit $] \rightarrow[$ Symbol $]$ corresponding to encode $_{2}$ is specified by

ss $\underline{\text { begins }}$ decode $e_{2} m$ int (encode $2 m$ int $s s$ )

for all finite sequences of symbols ss.

To implement decode $e_{2}$ we have somehow to invert streams. We will make use of a function destream with type

$$
\begin{aligned}
\text { destream }:: & (\text { state } \rightarrow \text { Maybe }(\text { output }, \text { state })) \rightarrow \\
& (\text { state } \rightarrow \text { input } \rightarrow \text { state }) \rightarrow \\
& (\text { state } \rightarrow[\text { output }] \rightarrow \text { input }) \rightarrow \\
& \text { state } \rightarrow[\text { output }] \rightarrow[\text { input }]
\end{aligned}
$$

The definition of destream is

$$
\begin{aligned}
& \text { destream } f g h z \text { ys } \\
& \text { case } f z \text { of } \\
& \quad \text { Just }\left(y, z^{\prime}\right) \rightarrow \text { destream } f g h z^{\prime}(\text { ys after } y) \\
& \quad \text { Nothing } \rightarrow x: \text { destream } f g h(g z x) y s \\
& \text { where } x=h z \text { ys }
\end{aligned}
$$

The operator after is partial:

$$
\text { ys after } y=\text { if head } y s=y \text { then tail ys else } \perp
$$

The function destream is dual to stream: when $f z$ produces something, an element of the input is consumed; when $f z$ produces nothing, an element of the output is produced using the helper function $h$. Note that destream always produces a partial or infinite list, never a finite one.

The relationship between stream and destream is given by the following theorem:

Theorem 35. Suppose the following implication holds for all $z, x$ and $x s$ :

$$
f z=\text { Nothing } \Rightarrow h z(\text { stream } f g z(x: x s))=x
$$

Then, provided stream $f g z$ xs returns a finite list, we have

$$
x s \text { begins destream } f g h z(\text { stream } f g z x s)
$$

Proof. The proof is by a double induction on $x s$ and $n$, where $n$ is the length of stream $f g z x s$.

Case []: Immediate since [] begins every list. 
Case $x: x s$ : We first consider the subcase $f z=$ Nothing (which includes the case $n=0)$ :

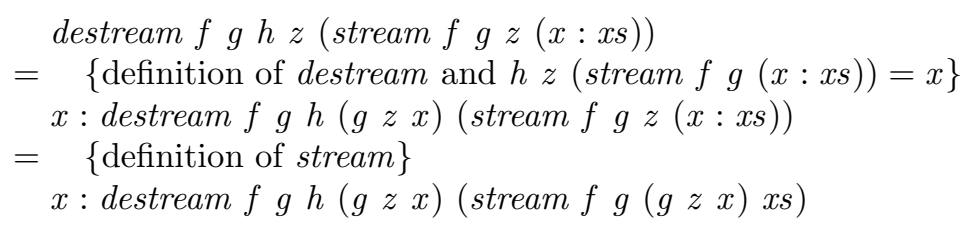

Since $(x: x s) \underline{\text { begins }}\left(x: x s^{\prime}\right)$ if and only if $x s \underline{\text { begins }} x s^{\prime}$, an appeal to induction establishes the case.

In the case $f z=\operatorname{Just}\left(y, z^{\prime}\right)$, we have $n \neq 0$, and so stream $f g z^{\prime}(x: x s)$ has length $n-1$. We reason

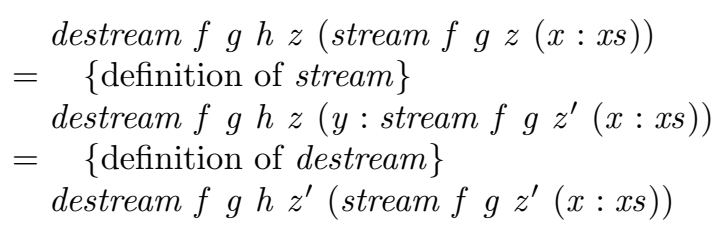

An appeal to induction establishes the case, completing the proof.

\subsection{Applying the theorem}

In order to apply the stream inversion theorem, recall Lemma 10 which states that foldl $(\triangleright)$ int $\cdot$ encodeSyms $m=$ snd $\cdot$ foldl step $(m$, int $)$ where

$$
\text { step }(m, \text { int }) s=(\text { newModel } m s \text {, int } \triangleright \text { encodeSym } m s)
$$

This identity allows us to fuse encodeSyms into the narrowing process:

$$
\text { encode }_{2} m \text { int }=\text { unfoldr nextBitM } \cdot \text { foldl step }(m, \text { int })
$$

where nextBitM is identical to nextBit except that it propagates the model as an additional argument:

$$
\begin{array}{ll}
\text { nextBitM } & ::(\text { Model }, \text { Interval }) \rightarrow \text { Maybe }(\text { Bit },(\text { Model }, \text { Interval })) \\
\operatorname{nextBitM~}(m,(l, r)) & \\
\mid r \leq 1 / 2 & =\text { Just }(0,(m,(2 \times l, 2 \times r))) \\
1 / 2 \leq l & =\text { Just }(1,(m,(2 \times l-1,2 \times r-1))) \\
\mid \text { otherwise } & =\text { Nothing }
\end{array}
$$

Theorem 30 is again applicable and we obtain the following alternative definition of encode $_{2}$ :

$$
\text { encode }_{2} m \text { int }=\text { stream nextBitM step }(m, \text { int })
$$

Now we are ready for stream inversion. Observe that encode $e_{2} m$ int returns a finite bit sequence on all finite symbol sequences, so it remains to determine $h$. 
Let $b s=$ encode $_{2} m$ int $(s: s s)$ and $x=$ fromBits $b s$, so that

$x \underline{\text { within }}$ (int $\triangleright$ encodeSym $m s$ )

We can now reason:

$$
\begin{aligned}
& x \underline{\text { within }} \text { (int } \triangleright \text { encodeSym } m s \text { ) } \\
& \equiv \quad\{\text { with } \text { int }=(l, r) \text { and encodeSym } m s=(p, q)\} \\
& l+(r-l) \times p \leq x<l+(r-l) \times q \\
& \equiv\{\text { arithmetic }\} \\
& p \leq(x-l) /(r-l)<q \\
& \equiv \quad\{\text { definition of decodeSym }\} \\
& s=\text { decodeSym } m((x-l) /(r-l))
\end{aligned}
$$

Hence we can take

$$
h(m,(l, r)) b s=\text { decodeSym } m((\text { fromBits bs }-l) /(r-l))
$$

Putting these pieces together, we therefore obtain

$$
\begin{array}{ll}
\text { decode }_{2} m \text { int } & =\text { destream nextBitM step nextSym }(m, \text { int }) \\
\text { nextSym }(m,(l, r)) \text { bs } & =\text { decodeSym } m((\text { fromBits bs }-l) /(r-l)) \\
\text { step }(m, \text { int }) s & =(\text { newModel } m s, \text { int } \triangleright \text { encodeSym } m s)
\end{array}
$$

where nextBitM was defined above.

This is not a very efficient way to compute decode $e_{2}$. Each computation of fromBits $b s$ requires that the bit sequence $b s$ is traversed in its entirety. Worse, this happens each time an output symbol is produced. Better is to fuse the computation of fromBits into destream so that the bit sequence is processed only once. We can do this fusion with a somewhat more complicated version of destream.

\subsection{A better stream inversion theorem}

Replace the previous function destream with a more general one, called unstream, with type

$$
\begin{aligned}
\text { unstream }:: & (\text { state } \rightarrow \text { Maybe }(\text { output }, \text { state })) \rightarrow \\
& (\text { state } \rightarrow \text { input } \rightarrow \text { state }) \rightarrow \\
& (\text { state } \rightarrow \text { result } \rightarrow \text { input }) \rightarrow \\
& (\text { result } \rightarrow \text { output } \rightarrow \text { result }) \rightarrow \\
& \text { state } \rightarrow \text { result } \rightarrow[\text { input }]
\end{aligned}
$$

With six arguments this seems a complicated function, which is why we didn't give it earlier. The definition of unstream is

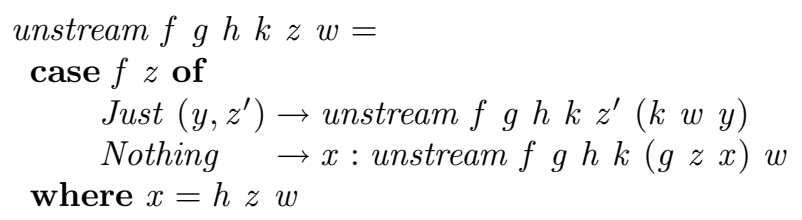


This more complicated definition is a generalisation, since destream $f g h z$ is equivalent to unstream $f g h$ after $z$. The relationship between stream and unstream is given by the following theorem, a generalisation of Theorem 35:

Theorem 36. Let process $z=$ foldr $(\oplus) w \cdot$ stream $f g z$. Suppose that

$$
f z=\text { Nothing } \Rightarrow h z \text { (process } z(x: x s))=x
$$

for all $z, x$ and $x s$. Furthermore, suppose that $\ominus$ satisfies $(y \oplus w) \ominus y=w$ for all $y$ and $w$. Then, provided stream $f g z$ xs returns a finite list, we have

$x s$ begins unstream $f g h(\ominus) z$ (process $z$ xs)

The proof is so similar to the earlier one that we can leave details as an exercise. The point of the new version is that, since fromBits $=$ foldr pack $(1 / 2)$ where pack $b x=(b+x) / 2$, we can define $\ominus=$ unpack, where unpack $x b=$ $2 \times x-b$. As a consequence, we obtain

$$
\begin{aligned}
& \text { decode }_{2} m \text { int } b s= \\
& \text { unstream nextBitM step nextSym unpack ( } m \text {, int) (fromBits bs) }
\end{aligned}
$$

In this version the bit sequence $b s$ is traversed only once. Nevertheless, decode $e_{2}$ is not an incremental algorithm since all of $b s$ has to be inspected before any output is produced.

Exercise 3\%. Following the steps of the proof of the first version of stream inversion, prove the second version of stream inversion.

Exercise 38. What substitutions for $\oplus$ and $w$ in Theorem 36 yield Theorem 35 ?

\section{Interval expansion}

The major problem with encode $e_{2}$ and decode $_{2}$ is that they make use of fractional arithmetic. In Section 8 we are going to replace fractional arithmetic by arithmetic with limited-precision integers. In order to do so we need a preparatory step: interval expansion. Quoting from Howard and Vitter [8]:

The idea is to prevent the current interval from narrowing too much when the endpoints are close to $1 / 2$ but straddle $1 / 2$. In that case we do not yet know the next output bit, but we do know that whatever it is, the following bit will have the opposite value; we merely keep track of that fact, and expand the current interval about $1 / 2$. This follow-on procedure may be repeated any number of times, so the current interval is always strictly longer than $1 / 4$.

For the moment we will just accept the fact that ensuring the width of the current interval is greater than $1 / 4$ before narrowing is an important step on the path to limited precision. 
Formally, interval expansion is a data refinement in which an interval $(l, r)$ is represented by a triple of the form $\left(n,\left(l^{\prime}, r^{\prime}\right)\right)$ satisfying

$$
l^{\prime}=\text { scale }(n, l) \text { and } r^{\prime}=\text { scale }(n, r)
$$

where scale $(n, x)=2^{n} \times(x-1 / 2)+1 / 2$, subject to $0 \leq l^{\prime}<r^{\prime} \leq 1$. In particular, $(0,(l, r))$ is one possible representation of $(l, r)$.

A fully-expanded interval for $(l, r)$ is a triple $\left(n,\left(l^{\prime}, r^{\prime}\right)\right)$ in which $n$ is as large as possible. Intervals straddling $1 / 2$ will be fully-expanded immediately before narrowing. The remainder of this section is devoted to installing this data refinement. More precisely, with ei denoting an expanded interval and contract ei the corresponding un-expanded interval, our aim is to provide suitable definitions that justify the following calculation:

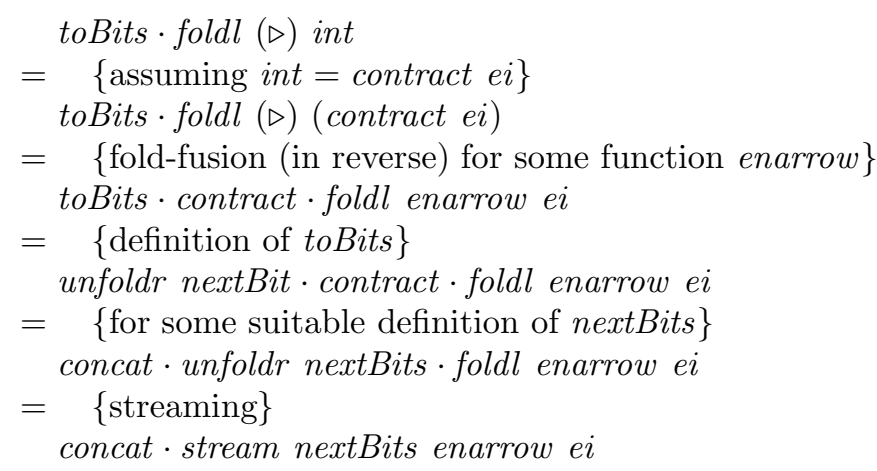

The function enarrow connotes "expand and narrow" and is an operation that first expands an interval before narrowing it. Given this motivating calculation, we can then define

encode $_{3} m$ ei $=$ concat $\cdot$ stream nextBits enarrow ei $\cdot$ encodeSyms $m$

Arithmetic coding is then implemented by the call encode $3(0,(0,1))$. Note that composing concat with stream still gives incremental transmission because of laziness: the argument to concat does not have to be evaluated fully before results are produced.

\subsection{Defining expand and contract}

First, we give a definition of the function expand that expands intervals. Observe that

$$
\begin{aligned}
& 0 \leq 2 \times(l-1 / 2)+1 / 2 \equiv 1 / 4 \leq l \\
& 2 \times(r-1 / 2)+1 / 2 \leq 1 \equiv r \leq 3 / 4
\end{aligned}
$$

Hence we can further expand $(n,(l, r))$ if $1 / 4 \leq l$ and $r \leq 3 / 4$. This leads to the definition

$$
\begin{aligned}
& \text { expand }(n,(l, r)) \\
& \qquad 1 / 4 \leq l \wedge r \leq 3 / 4=\text { expand }(n+1,(2 \times l-1 / 2,2 \times r-1 / 2)) \\
& \mid \text { otherwise }=(n,(l, r))
\end{aligned}
$$


The function nextBits, to be defined in a short while, will return Nothing on intervals that straddle $1 / 2$. Consequently, in encode 3 we expand intervals $(l, r)$ satisfying $l<1 / 2<r$ immediately before narrowing. It follows that narrowing is applied only when $l<1 / 4$ and $1 / 2<r$, or $l<1 / 2$ and $3 / 4<r$; in either case, $1 / 4<r-l$, which is the key inequality.

The converse of expand is given by

$$
\text { contract }(n,(l, r))=(\text { rescale }(n, l), \text { rescale }(n, r))
$$

where rescale $(n, x)=(x-1 / 2) / 2^{n}+1 / 2$. We leave it as exercises to verify that

$$
\begin{array}{ll}
\text { contract } \cdot \text { expand } & =\text { contract } \\
\text { contract }(n, \text { int } 1 \triangleright \text { int } 2) & =\text { contract }(n, \text { int } 1) \triangleright \text { int } 2
\end{array}
$$

Consequently, defining enarrow by

$$
\begin{aligned}
\text { enarrow ei int } 2= & (n, \text { int } 1 \triangleright \text { int } 2) \\
& \text { where }(n, \text { int } 1)=\text { expand } e i
\end{aligned}
$$

we have contract $($ enarrow ei int $)=$ contract ei $\triangleright$ int. An appeal to fold-fusion therefore gives

$$
\text { contract } \cdot \text { foldl enarrow } \mathrm{ei}=\text { foldl }(\triangleright)(\text { contract ei) }
$$

This identity was used in the motivating calculation above. The remaining step is to find some suitable definition of nextBits so that

$$
\text { toBits } \cdot \text { contract }=\text { concat } \cdot \text { unfoldr nextBits }
$$

and also that nextBits and enarrow satisfy the streaming condition.

The definition of nextBits turns out to be

$$
\begin{aligned}
\text { nextBits }(n,(l, r)) & \\
\mid r \leq 1 / 2 & =\text { Just }(\text { bits } n 0,(0,(2 \times l, 2 \times r))) \\
\mid 1 / 2 \leq l & =\text { Just }(\text { bits } n 1,(0,(2 \times l-1,2 \times r-1))) \\
\mid \text { otherwise } & \text { Nothing }
\end{aligned}
$$

where bits $n b=b$ : replicate $n(1-b)$ returns a $b$ followed by a sequence of $n$ copies of $1-b$. The proof that this definition satisfies all our requirements is left as an exercise.

Exercise 39. Verify that

$$
\begin{aligned}
\text { contract } \cdot \text { expand } & =\text { contract } \\
\text { contract }(n, \text { int } 1 \triangleright \text { int } 2) & =\text { contract }(n, \text { int } 1) \triangleright \text { int } 2
\end{aligned}
$$

Why don't we have contract $\cdot$ expand $=i d$ ?

\section{Exercise 40. Prove that}

$$
\begin{aligned}
& \text { rescale }(n, x) \leq 1 / 2 \equiv x \leq 1 / 2 \\
& \text { rescale }(n, x) \geq 1 / 2 \equiv x \geq 1 / 2
\end{aligned}
$$

Hence contract $(n,(l, r))$ straddles $1 / 2$ iff $(l, r)$ does. 
Exercise 41. Prove that

$$
\begin{aligned}
& 2 \times \text { rescale }(n+1, x)=\text { rescale }(n, x)+1 / 2 \\
& 2 \times \text { rescale }(n+1, x)-1=\text { rescale }(n, x)-1 / 2
\end{aligned}
$$

Exercise 42. Prove by induction on $n$ that

$$
\begin{aligned}
& \text { toBits }(2 \times \text { rescale }(n, l), 2 \times \text { rescale }(n, r))= \\
& \quad=\text { replicate } n 1+\text { toBits }(2 \times l, 2 \times r) \\
& \text { toBits }(2 \times \text { rescale }(n, l)-1,2 \times \text { rescale }(n, r)-1)= \\
& \quad=\text { replicate } n 0+\text { toBits }(2 \times l-1,2 \times r-1)
\end{aligned}
$$

Exercise 43. Prove that if $l<1 / 2<r$ then

$$
\text { toBits }(\text { contract }(n,(l, r)))=\text { concat }(\text { unfoldr nextBits }(n,(l, r)))
$$

Exercise 44. Prove that if $r \leq 1 / 2$ then

$$
\text { toBits }(\text { contract }(n,(l, r)))=\text { bits } n 0+\text { toBits }(2 \times l, 2 \times r)
$$

Similarly, prove that if $1 / 2 \leq l$ then

$$
\text { toBits }(\text { contract }(n,(l, r)))=\text { bits } n 1+\text { toBits }(2 \times l-1,2 \times r-1)
$$

Hence complete the proof of toBits $\cdot$ contract $=$ concat $\cdot$ unfoldr nextBits.

Exercise 45. Verify that the streaming condition holds for nextBits and enarrow.

\section{From fractions to integers}

We now want to replace fractional arithmetic by arithmetic with limited-precision integers. In the final version of arithmetic coding, intervals take the form $(l, r)$, where $l$ and $r$ are integers in the range $0 \leq l<r \leq w$ and $w$ is a fixed power of two. This pair represents the interval $(l / w, r / w)$.

Intervals in each model $m$ take the form $(p, q, d)$, where $p$ and $q$ are integers in the range $0 \leq p<q \leq d$ and $d$ is an integer which is fixed for $m$ and called the denominator for $m$. This triple represents the interval $(p / d, q / d)$.

\subsection{Integer narrowing}

The narrowing function is redefined as follows:

$$
(l, r)>(p, q, d)=(l+\lfloor(r-l) \times p / d\rfloor, l+\lfloor(r-l) \times q / d\rfloor)
$$

Equivalently,

$$
(l, r) \triangleright(p, q, d)=(l+((r-l) \times p) \underline{d i v} d, l+((r-l) \times q) \underline{d i v} d)
$$

A reasonable step, you might think, but there are a number of problems with it: 
- the revised definition of narrowing completely changes the specification: encoding will now produce different outputs than before and, in general, the effectiveness of compression will be reduced;

- worse, $\boldsymbol{\sim}$ is not associative, and none of the foregoing development applies;

- unless we take steps to avoid it, intervals can collapse to the empty interval when $\left\lfloor(r-l) \times{ }^{p} / d\right\rfloor=\left\lfloor(r-l) \times{ }^{q} / d\right\rfloor$.

The middle point seems the most damaging one, and is perhaps the reason that writers on arithmetic coding do not attempt to specify what problem arithmetic coding solves.

\subsection{Change of specification}

Fortunately, we can recover all of the previous development. Observe that

$$
(l, r) \triangleright(p, q, d)=(l / w, r / w) \triangleright\left(p^{\prime} / d,{ }^{\prime} / d\right)
$$

where

$$
\begin{aligned}
& p^{\prime}=d / r-l \times\lfloor(r-l) \times p / d\rfloor \\
& q^{\prime}=d / r-l \times\lfloor(r-l) \times q / d\rfloor
\end{aligned}
$$

Hence, provided $p^{\prime}<q^{\prime}$, integer narrowing of an interval $(l, r)$ by another interval $(p, q)$ drawn from a model $m$ can be viewed as fractional narrowing of $(l, r)$ by the corresponding interval $\left(p^{\prime}, q^{\prime}\right)$ drawn from an adjusted model adjust $(l, r) \mathrm{m}$. Note that $p^{\prime} \leq p$ and $q^{\prime} \leq q$, so the effect of this adjustment is that some of the intervals shuffle down a little, leaving a little headroom at the top (see below for an example). We do not need to implement adjust; the important point is by invoking it at every step all of the previous development remains valid.

It is instructive to illustrate the adjustments made to the model. Consider Figure 1 in which $w=64$ and $d=10$. The columns on the left show a given sequence of models that might arise after processing symbols in the string ABAC. For example, the first row shows a model in which $A$ is associated with the interval $[0.0 . .0 .3), B$ is associated with [0.3..0.6), and $C$ with [0.6..1.0). The columns on the right show the corresponding adjusted intervals to three decimal places. The current intervals immediately before processing the next symbol are shown in the middle. The output of the integer implementation is 0010010, while that of the real implementation is 00100 , so there is a deterioration in compression effectiveness even for this short string.

\subsection{When intervals collapse}

It is left as an exercise to show that

$$
(\forall p, q: 0 \leq p<q \leq d:\lfloor(r-l) \times p / d\rfloor<\lfloor(r-l) \times q / d\rfloor)
$$

if and only if $d \leq r-l$. Hence we have to ensure that the width of each interval is at least $d$ before narrowing. But interval expansion guarantees that the width 


\begin{tabular}{lccc|l|ccc} 
models & A & B & C & adjustments & A & B & C \\
\hline initial model: & 0.0 & 0.3 & 0.6 & adjust $(0,64):$ & 0.0 & 0.297 & 0.594 \\
after A: & 0.0 & 0.4 & 0.7 & adjust $(0,38):$ & 0.0 & 0.395 & 0.684 \\
after B: & 0.0 & 0.4 & 0.8 & adjust $(30,52):$ & 0.0 & 0.364 & 0.773 \\
after A: & 0.0 & 0.4 & 0.8 & adjust $(24,56):$ & 0.0 & 0.375 & 0.781 \\
after C: & 0.0 & 0.5 & 0.7 & adjust $(8,64):$ & 0.0 & 0.500 & 0.696
\end{tabular}

Fig. 1. Model adjustment

of each (expanded) interval is greater than $w / 4$ before narrowing, so interval collapse is avoided if $w / 4 \geq d$. That was the whole point of making use of interval expansion.

Since $w \times d \leq w \times w / 4=2^{2 \times e-2}$ if $w=2^{e}$, we have to ensure that our limited-precision arithmetic is accurate to $2 \times e-2$ bits.

Exercise 46. Prove that

$$
(\forall p, q: 0 \leq p<q \leq d:\lfloor(r-l) \times p / d\rfloor<\lfloor(r-l) \times q / d\rfloor)
$$

if and only if $d \leq r-l$.

Exercise 4\%. According to the Haskell Report [1], the finite-precision type Int covers at least the range $\left[-2^{29}, 2^{29}-1\right]$. What are suitable choices for $w$ and $d$ ?

\subsection{Final version of encode}

Gathering together the ingredients of this data refinement, we can now give the final version of encode:

$$
\text { encode } m \text { ei }=\text { concat } \cdot \text { stream nextBits enarrow ei } \cdot \text { encodeSyms } m
$$

where

$$
\begin{aligned}
& \text { enarrow ei int } 2 \quad=(n \text {, int } 1>\text { int } 2) \\
& \text { where }(n, \text { int } 1)=\text { expand ei } \\
& \text { expand }(n,(l, r)) \\
& { }^{w} / 4 \leq l \wedge r \leq 3 \times{ }^{w} / 4=\operatorname{expand}\left(n+1,\left(2 \times l-{ }^{w} / 2,2 \times r-{ }^{w} / 2\right)\right) \\
& \text { otherwise } \quad=(n,(l, r)) \\
& \text { nextBits }(n,(l, r)) \\
& \begin{array}{ll}
\mid r \leq w / 2 & =J u s t ~(b i t s ~ n 0,(0,(2 \times l, 2 \times r))) \\
w / 2 \leq l & =J u s t(\text { bits } n 1,(0,(2 \times l-w, 2 \times r-w))) \\
\text { otherwise } & \text { Nothing }
\end{array}
\end{aligned}
$$

Arithmetic coding is now implemented by encode $m(0,(0, w))$.

Exercise 48. Instead of using semi-open intervals [l..r $)$ we could use a closed interval $[l . . r-1]$. What modifications are required to the definitions of encode and decode, and why should such a representation have an advantage over the semi-open one? 
Exercise 49. Notwithstanding everything that has gone before, encoding is not guaranteed to work with any form of limited-precision arithmetic! Why not?

Exercise 50. Imagine a static model of three equiprobable symbols A, B and C, so that $B$ is assigned the range $\left[1 / 3 . .^{2} / 3\right)$. Suppose a message of a billion $B$ 's is to be encoded. What is the output? How big does $n$ get in the definition of expand? What does this relationship reveal about the answer to the previous exercise?

\subsection{Decoding in the integer version}

Decoding with limited-precision arithmetic is again implemented by appeal to stream inversion, just as in the previous version. Let us start by showing how to compute the symbol $s$ from $b s=$ encode $m$ ei $(s: s s)$ under the assumption that nextBits ei $=$ Nothing, so that $e i$ straddles $1 / 2$ and expand ei delivers an integer that will not collapse to the empty interval on narrowing. Setting wfromBits $=(w \times) \cdot$ fromBits, we know that $x=$ wfromBits bs is a fraction in the interval $[0 . . w)$ satisfying

$$
x \text { within contract (enarrow ei (encodeSym } m s) \text { ) }
$$

How can we compute $s$ given $x, m$, and $e i$ ? We need to be able to do this in order to define the helper function nextSym for unstream.

To determine $s$, we make use of the following property of floors: for all integers $n$ and fractions $x$, we have $n \leq\lfloor x\rfloor \equiv n \leq x$. Ignorance of this simple rule has marred practically every published paper on arithmetic coding that we have read.

We now reason:

$$
\begin{aligned}
& x \text { within (contract (enarrow ei (encodeSym } m s) \text { )) } \\
& \equiv \quad\{\text { setting }(n,(l, r))=\text { expand ei }\} \\
& x \underline{\text { within }}(\text { contract }(n,(l, r) \backslash \text { encodeSym } m s)) \\
& \equiv\{\text { setting } y=\text { scale }(n, x)\} \\
& y \underline{\text { within }}((l, r) \triangleright \text { encodeSym } m s) \\
& \equiv\{\text { setting }(p, q, d)=\text { encodeSym } m s\} \\
& l+\lfloor(r-l) \times p / d\rfloor \leq y<l+\lfloor(r-l) \times q / d\rfloor \\
& \equiv\{\text { arithmetic }\} \\
& \left\lfloor(r-l) \times{ }^{p} / d\right\rfloor \leq y-l<\lfloor(r-l) \times q / d\rfloor \\
& \equiv \quad \text { rule of floors, setting } k=\lfloor y\rfloor\} \\
& \left\lfloor(r-l) \times{ }^{p} / d\right\rfloor \leq k-l<\left\lfloor(r-l) \times{ }^{q} / d\right\rfloor \\
& \equiv\{\text { arithmetic }\} \\
& \left\lfloor(r-l) \times{ }^{p} / d\right\rfloor<k-l+1 \leq\lfloor(r-l) \times q / d\rfloor \\
& \equiv \text { \{rule of floors } \\
& (r-l) \times p / d<k-l+1 \leq(r-l) \times q / d \\
& \equiv\{\text { arithmetic }\} \\
& p \leq((k-l+1) \times d-1) /(r-l)<q \\
& \equiv \quad \text { rule of floors }\} \\
& p \leq\lfloor((k-l+1) \times d-1) /(r-l)\rfloor<q
\end{aligned}
$$


Hence, redefining decodeSym to have type $M o d e l \rightarrow I n t \rightarrow$ Symbol, we have

$$
\begin{aligned}
\operatorname{nextSym}(m, e i) x & =\text { decodeSym } m t \\
\text { where } t & =((k-l+1) \times \text { denom } m-1) \underline{\text { div }}(r-l) \\
k & =\lfloor\text { scale }(n, x)\rfloor \\
(n,(l, r)) & =\text { expand } e i
\end{aligned}
$$

Armed with this result, we can now tackle the task of inverting encode. First, as before, we rewrite encode in the form

$$
\text { encode } m \text { ei }=\text { concat } \cdot \text { stream nextBitsM step }(m, e i)
$$

where step $(m, e i) s=($ newModel $m$ s, enarrow ei (encodeSym $m s)$ ) and nextBits $M$ carries the model as an extra argument:

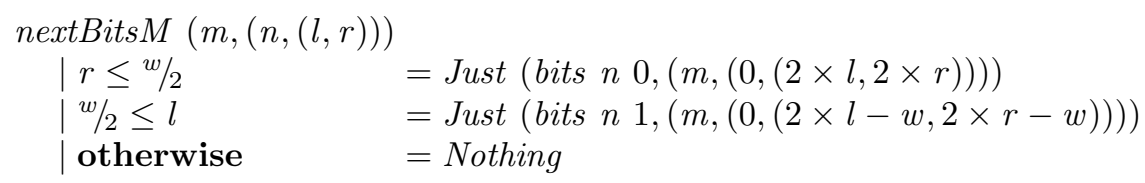

Now set $x=$ wfromBits (concat (stream nextBitM step $(m, e i)(s: s s)$ )). An appeal to fold-fusion gives

$$
\begin{aligned}
w \text { fromBits }= & \text { foldr pack }(w / 2) \\
& \text { where pack } b x=(w \times b+x) / 2
\end{aligned}
$$

A second appeal to fold-fusion gives

$$
\text { wfromBits } \cdot \text { concat }=\text { foldr }(\oplus)(w / 2)
$$

where $b s \oplus x=$ foldr pack $x$ bs. Moreover, defining

$$
x \ominus b s=\text { foldl unpack } x \text { bs }
$$

where unpack $x b=2 \times x-w \times b$, we have $(b s \oplus x) \ominus b s=x$ by Exercise 14 .

All the ingredients for destreaming are now in place, and we can define

$$
\begin{aligned}
& \text { decode } m \text { ei } b s= \\
& \quad \text { unstream nextBitsM step nextSym }(\ominus) \text { ( } m, \text { ei) (wfromBits bs) }
\end{aligned}
$$

where

$$
\begin{aligned}
& \text { nextSym }(m, e i) x=\text { decodeSym } m t \\
& \text { where } t=((k-l+1) \times \text { denom } m-1) \underline{\operatorname{div}}(r-l) \\
& k \quad=\lfloor\text { scale }(n, x)\rfloor \\
& (n,(l, r))=\text { expand } \text { ei }
\end{aligned}
$$

and

$$
\begin{aligned}
x \ominus b s= & \text { foldl unpack } x \text { bs } \\
& \text { where unpack } x \quad b=2 \times x-w \times b
\end{aligned}
$$

The one remaining fly in the ointment is that decode is not incremental, as all elements of $b s$ are inspected in order to compute wfromBits $b s$. 


\subsection{A final data refinement}

Consider the first invocation of nextSym in the computation decode $m$ ei bs. We have to compute

$$
k=\left\lfloor 2^{n} \times(\text { wfromBits } b s-w / 2)+w / 2\right\rfloor
$$

This can be done without inspecting all of $b s$. We only need to compute the first $e+n$ bits, where $w=2^{e}$. This is the clue to making decode incremental.

Suppose we represent $b s$ not by $x=$ wfromBits $b s$ but by a pair $(z, r s)$ where $z$ is the binary integer formed from take $e$ bs (assuming $b s$ contains at least $e$ bits) and $r s=d r o p$ e bs. Then $z=\lfloor$ wfromBits $b s\rfloor$. If $b s$ contains fewer than $e$ bits, then we can always append a 1 to $b s$ followed by a sufficent number of 0s. To justify this, recall Exercise 20. Let us call this computation buffering and write $(z, r s)=$ buffer bs.

Given $(z, r s)=$ buffer $b s$ we can now compute $k=$ fscale $(n,(z, r s))$, where

$$
\text { fscale }(n,(z, r s))=\text { foldl }\left(\lambda x b \rightarrow 2 \times x+b-{ }^{w} / 2\right) z(\text { take } n \text { rs })
$$

The proof is left as an exercise. Hence $k$ can be computed by inspecting only the first $e+n$ bits of $b s$.

To install this final refinement we need to show how to compute buffer. There are two ways to do it and we will need both. The first is to define

$$
\begin{aligned}
\text { buffer bs }= & (\text { foldl }(\lambda x b \rightarrow 2 \times x+b) 0 c s, r s) \\
& \text { where }(c s, r s)=\operatorname{splitAt} e(b s+1: \text { replicate }(e-1) 0)
\end{aligned}
$$

The definition of $z$ uses the standard method for converting a bit string into a binary integer. This method is used in the final version of decode.

But we also have to show how to maintain the representation $(z, r s)$ during the destreaming process. We leave it as an exercise to show that buffer can also be computed by

$$
\begin{aligned}
\text { buffer }= & \text { foldr op }(w / 2,[]) \text { bs } \\
\text { op } b(z, r s)= & (y, r: r s) \\
& \text { where }(y, r)=(w \times b+z) \underline{\text { divMod } 2}
\end{aligned}
$$

The point of this alternative is that we have

$$
\text { foldr op }(w / 2,[]) \cdot \text { concat }=\text { foldr }(\oplus)(w / 2,[])
$$

where $b s \oplus(x, d s)=$ foldr op $(x, d s)$ bs. Moreover, we can invert $\oplus$ by defining $\ominus$ to be

$$
\begin{aligned}
& (z, r s) \ominus b s=\text { foldl unop }(z, r s) \text { bs } \\
& \text { unop }(z, r s) b=(2 \times z-w \times b+\text { head } r s, \text { tail } r s)
\end{aligned}
$$

Now all the ingredients for destreaming are once again in place.

Exercise 51. Show that $\lfloor$ scale $(n$, wfromBits bs $)\rfloor=$ fscale $(n$, buffer bs), where

$$
\text { fscale }(n,(z, r s))=\text { foldl }(\lambda x b \rightarrow 2 \times x+b-w / 2) z \text { (take } n r s)
$$


Exercise 52. Show that

$$
\begin{aligned}
\text { buffer }= & \text { foldr op }(w / 2,[]) \text { bs } \\
\text { op } b(z, r s)= & (y, r: r s) \\
& \text { where }(y, r)=(w \times b+z) \underline{\text { divMod } 2}
\end{aligned}
$$

\subsection{Final version of decode}

Here is the final version of decode:

$$
\begin{aligned}
& \text { decode } m \text { ei } b s= \\
& \text { unstream nextBitsM step nextSym }(\ominus) \text { ( } m, \text { ei) (buffer bs) } \\
& \text { buffer } b s=(z, r s) \\
& \text { where } z \quad=\text { foldl }(\lambda x b \rightarrow 2 \times x+b) 0 \text { cs } \\
& (c s, r s)=\text { splitAt } e(b s+1 \text { : replicate }(e-1) 0) \\
& \text { nextSym }(m, e i)(z, r s)=\text { decodeSym } m t \\
& \text { where } t=((k-l+1) \times \text { denom } m-1) \underline{\text { div }}(r-l) \\
& k \quad=\text { fscale }(n,(z, r s)) \\
& (n,(l, r))=\text { expand } e i \\
& (z, r s) \ominus b s=\text { foldl unop }(z, r s) b s \\
& \text { where unop }(z, r s) b=(2 \times z-w \times b+\text { head rs, tail } r s) \\
& \text { fscale } \left.(n,(z, r s))=\text { foldl }\left(\lambda x b \rightarrow 2 \times x+b-{ }^{w} / 2\right) z \text { (take } n \text { rs }\right)
\end{aligned}
$$

The remaining functions nextBits $M$, step, and expand were defined previously.

\section{Conclusions}

The reader who has followed us up to now will appreciate that there is rather a lot of arithmetic in arithmetic coding, and that includes the arithmetic of folds and unfolds as well as numbers. As we said at the start, arithmetic coding is a simple idea but one that requires care to implement with limited-precision integer arithmetic. To the best of our knowledge, no previous description of arithmetic coding has ever tackled the formal basis for why the method works, let alone providing a formal development of the coding and decoding algorithms.

Perhaps not surprisingly we went through many iterations of the development, considering different ways of expressing the concepts of streaming and stream inversion. The final constructions given above differ markedly from the versions given in the Summer School in August, 2002. None of these iterations would have been possible without the availability of a functional perspective, whose smooth proof theory enabled us to formulate theorems, prove them, and perhaps discard them, quite quickly. Whether or not the reader has followed all the details, we hope we have demonstrated that functional programming and equational reasoning are essential tools of thought for expressing and proving properties of complicated algorithms, and that the ability to define structured recursion operators, such as foldl, unfoldr, stream and destream, is critical for formulating and understanding patterns of computation. 


\section{References}

1. Haskell 98: A Non-Strict, Purely Functional Language. Available online at www.haskell.org/onlinereport.

2. Standard Libraries for Haskell 98. Available online at www.haskell.org/onlinelibrary.

3. R. S. Bird. Introduction to Functional Programming using Haskell. International Series in Computer Science. Prentice Hall, 1998.

4. R. S. Bird and O. de Moor. Algebra of Programming. International Series in Computer Science. Prentice Hall, 1997.

5. R. M. Fano. Transmission of Information. MIT Press, Cambridge MA, and Wiley, NY, 1961.

6. J. Gibbons. Origami programming. In The Fun of Programming, J. Gibbons and O. de Moor, eds, Palgrave, 2003.

7. J. Gibbons. The Third Homomorphism Theorem. J. Functional Prog., Vol 6 , No 4, 657-665, 1996.

8. P. G. Howard and J. S. Vitter. Arithmetic coding for data compression. Proc. IEEE, Vol 82, No 6, 857-865, 1994.

9. D. A. Huffman. A method for the construction of minimum-redundancy codes. Proc. Inst. Radio Eng. Vol 40, No 9, 1098-1101, Sept. 1952.

10. J. Jiang. Novel design of arithmetic coding for data compression. IEE Proc. Comput. Dig. Tech., Vol 142, 6 (Nov) 419-424, 1995.

11. E. Meijer, M. Fokkinga and R. Paterson. Functional programming with bananas, lenses, envelopes and barbed wire. In Functional Programming Languages and Computer Architecture, Lecture Notes in Computer Science 523, 124-144, 1991.

12. A. Moffat, R. M. Neal, and I. H. Witten. Arithmetic coding revisited. ACM Trans. on Inf. Systems Vol 16, No 3, 256-294, July 1998.

13. C. E. Shannon. A mathematical theory of communication. Bell Syst. Tech. J. Vol 27, 79-423, 1948.

14. I. H. Witten, R. M. Neal, and J. G. Cleary. Arithmetic coding for data compression. C. ACM, Vol 30, No 6, 520-540, June 1987. 